\title{
A Bayesian and Smart Gateway Based Communication For Noisy IoT Scenario
}

\author{
Cristanel Razafimandimby*, Valeria Loscrí ${ }^{*}$ Anna Maria Vegni ${ }^{\dagger}$, and Alessandro Neri ${ }^{\dagger}$ \\ ${ }^{*}$ Inria Lille - Nord Europe, Lille, France \\ Emails: \{jean.razafimandimby_anjalalaina,valeria.loscri\}@inria.fr \\ ${ }^{\dagger}$ Department of Engineering, Roma Tre University \\ COMLAB Telecommunication Laboratory, Rome, Italy \\ Emails: \{annamaria.vegni, alessandro.neri\}@uniroma3.it
}

\begin{abstract}
Nowadays, Internet of Things (IoT) coupled with cloud computing begins to take an important place in economic systems and in society daily life. It has got a large success in several application areas, ranging from smart city applications to smart grids. One major challenge that should be addressed is the huge amount of data generated by the sensing devices, which make the control of sending useless data very important.
\end{abstract}

To face this challenge, we present a Bayesian Inference Approach (BIA), which allows avoiding the transmission of high spatio-temporal correlated data. In this paper, BIA is based on a hierarchical architecture with simple nodes, smart gateways and data centers. Belief Propagation algorithm has been chosen for performing an approximate inference on our model in order to reconstruct the missing sensing data. BIA is evaluated based on the data collected from real sensors and according to different scenarios. The results show that our proposed approach reduces drastically the number of transmitted data and the energy consumption, while maintaining an acceptable level of data prediction accuracy.

Keywords-IoT, Belief Propagation, Markov Random Fields, Cloud, Smart Gateway.

\section{INTRODUCTION}

In recent years, Internet has become the main vector of information sharing. It took an important place not only in our economic systems, but also in our daily life. However, beyond the interconnection of computers and mobile terminals, its expansion capabilities are still significant since it is the enabler of the interactions among a large number of heterogeneous devices. The sensors are typical examples of such devices. This new extension of Internet is called Internet of Things (IoT), connecting, in addition of billions of human beings, also tens of billions of objects [6]. Moreover, areas that were relatively unaffected by the Internet (such as automotive, eletric power distribution, agriculture, etc.) will be devastated by this network extension [3], [10], [12], [13], [14].

Nevertheless, IoT raises yet many challenges and one of them is the management of massive amount of data generated by sensing devices. Storing this big data locally and even temporarily will not be possible any more. Therefore, harnessing cloud computing capacity is needed, but unfortunately this is not enough. However, it was observed that, with the increase of sensor density, data generated by IoT devices tend to be highly redundant. Thus, uploading raw data to the cloud can become extremely inefficient due to the waste of memory and network overloading.
To address this issue, either the IoT devices should avoid the generation of useless data or the gateway device should be able to stop uploading of redundant data from some devices, to reduce consumption of network and cloud resources.

From the above considerations, in this paper we present a Bayesian Inference Approach (BIA), which allows to remove a great amount of spatio-temporal correlation data in an IoT domain. BIA is based on Pearl's Belief Propagation (BP) algorithm that is an iterative technique mostly used for solving inference problems [20]. In the IoT context, the belief of a device is related to the physical quantity measured by the device sensors. BP infers the measurements of other neighboring nodes, especially in cases where the data are missing. In BPbased approaches, each node determines its belief by merging its local measurement with the beliefs of its neighboring nodes, and its beliefs obtained in the past run. BP provides therefore a spatio-temporal cooperation among several devices. A good correlation between data is important in such inference problems since it dictates the accuracy of data inference, and hence reduces the estimation error of the global information.

The main contributions of this paper can be summarized as follows:

- Adoption of a Bayesian Inference Approach that allows avoiding the transmission of useless data in heterogeneous IoT networks. The BP algorithm has been chosen to infer the missing data;

- Use of smart gateway based communications in order to decrease the estimation error in the cloud;

- Performance assessment based on data collected from real sensors.

This paper is organized as follows. Section II provides some related works on data propagation mechanisms in IoT context. Section III describes our reference cloudbased architecture for the IoT scenario. In Section IV, we present the proposed Bayesian Inference Approach based on BP algorithm for data sharing in a noisy IoT scenario. Section V provides the experimental results for the assessment of the proposed BIA technique in different real scenarios. Finally, conclusions are drawn at the end of the paper. 


\section{RELATED WORK}

In this section, we present several works closely related to ours. In order to clarify the difference of our work with those existing in the literature, we classify the related works according to the fields where they were treated, i.e., $(i)$ in Wireless Sensor Networks (WSNs), and (ii) IoT domain.

\section{A. Wireless Sensor Networks context}

Energy saving is considered as the most important part in WSNs, since sensors have to operate on extremely limited energy budget. A good survey of energy saving in WSNs context was proposed in [1]. Data reduction is one of best approaches to achieve energy-efficiency in data acquisition. Its main goal is the reduction of the amount data to be transmitted over the network such that the energy spent for communications decreases. Many data prediction approaches have been proposed in several works [9], [15], [16], [21]. In [19], the authors have combined data prediction and lossy compression scheme to jointly achieve accuracy and efficiency of the data processing in clustered WSNs.

Even if many approaches have been developed for data prediction in the WSNs context, all the proposed approaches deal only with homogeneous data. However, the heterogeneity of IoT devices requires that the employed prediction model has be applicable to a variety of applications with heterogeneous data. Moreover, the prediction models used in the WSNs context are often too computationally expensive to run or do not provide room for further data reduction. All these points make many existing methods in WSNs unsuitable for the IoT context.

\section{B. IoT context}

One of the most distinguishing aspects of the IoT is the fact that the data are acquired from a variety of sources. As far as we know, there exists only two works that take in account this heterogeneous aspect of IoT. The first work was proposed in [4], and the other was proposed in [17].

In [4], the authors presented a cloud-based Adaptive Sensing Belief Propagation (ASBP) protocol with energyefficient data fusion for IoT applications. Our work differs from [4] in the following aspects :

- We assume that the correlations between different kind of data (e.g. temperature, humidity, light and voltage) are a priori known. Consequently, we do not need to compute the correlation matrix each time;

- The knowledge of the correlation is used only to decide what data have to be sent or inferred in the cloud. Therefore, we do not operate the sensor selection optimization problem in this work;

- We propose a smart gateway concept in order to decrease the inference error in the cloud. Hence, we provide a dual prediction scheme: one in the gateway, and the other in the cloud. Both predictions are based on the BP algorithm.

Then, the authors of [17] in turn presented a theoretical bayesian based approach for data sharing in the

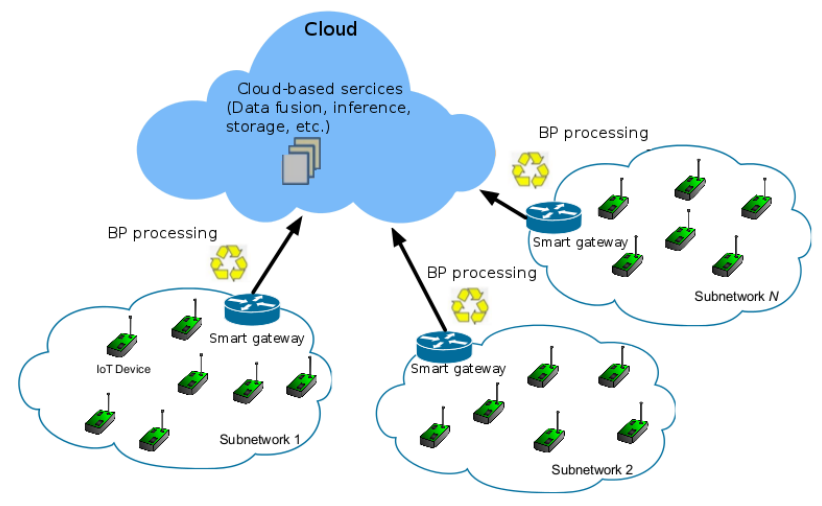

Figure 1: A cloud-based IoT network model.

IoT context. In this paper, we go a step further than [17] by proposing an extensive experimentation scenario and smart gateway based communications.

\section{NETWORK MODEL}

As depicted in Figure 1, in this paper we propose a BP approach in a cloud-based architecture consisting of simple nodes, smart gateways and data centers. Each entity in our architecture plays a different role w.r.t the functionalities, the computational and communication capabilities. Our IoT network model may include multiple subnets associated with different applications. Each subnet is composed of IoT devices connected to each others for data sharing, and a smart gateway that relays the data flows to the cloud. The cloud in turn is responsible of inference, storage and all the cloud-based services.

In a given IoT application, the sensor nodes periodically collect environmental data, such as temperature, humidity and illuminance, and forward them to the gateways using a multi-hop routing protocol. Then, the gateways collect the data and decide what has to be sent to the cloud. This decision depends on the fact that the gateway knows or not the priori probability of inference error of the used approach.

Figure 4 shows an example of data considered in our IoT applications. These data are a subset of the data collected from 54 sensors deployed in the Intel Berkeley Research lab [11]. This figure depicts precisely the correlation between the temperature, humidity, light and voltage data. As we can noticed, there is a good correlation between temperature and humidity data. Indeed, it is really easy to draw the regression line between temperature and humidity data here. Therefore, it will be more efficient to infer temperature value from humidity value or conversely (i.e., infer humidity value from temperature value).

\section{BAYESIAN INFERENCE APPROACH}

In this section, we describe our BIA technique. As mentioned before, our main goal is to avoid sending useless data, while keeping an acceptable level of data content accuracy. BIA is based on Pearl's BP algorithm that will be described below. 


\section{A. Model}

As a starting point before any inference procedure, the design of a graphical model should be provided. Graphical models are schematic representations of probability distributions [5]. They consist of nodes connected by either directed or undirected edges. Each node represents a random variable, and the edges represent probabilistic relationships between variables. Models which are comprised of directed edges are known as Bayesian networks, whilst models that are composed of undirected edges are known as Markov random fields (MRF) [18]. As in [17], we present an inference approach under the hypothesis of MRF, modeled by means of Factor Graphs. It follows that our goal is to estimate the state $X$ of the sensed environment starting from the sets of data collected by each sensor node. If $X$ is modeled as a MRF, by taking advantage of the Hammersley-Clifford Theorem, the joint probability distribution of $X$ with a set of cliques ${ }^{1} C$ is given by

$$
p_{X}(x)=\frac{1}{Z} \exp \{-E(x)\},
$$

where $Z$ is the normalization factor, and

$$
E(x)=\sum_{c \in C} \psi_{c}\left(x_{c}\right)
$$

is the energy function, $\psi_{c}$ is the potential associated with clique $c \in C$, and $x_{c}$ is the set of nodes belonging to the clique indexed by $c$.

\section{B. Belief Propagation}

Once the model has been defined, queries can be performed on the model to find the marginal probability distribution for one node or a set of nodes in the network graph. We use BP algorithm for this purpose and also for the computation cost reason. BP is a well known algorithm for performing inference on graphical models [20]. In general, we assume that some observations are made and some other data about the underlying environment will be inferred. The choice of data to infer is based on the strong correlation between data.

Given the $i$-th device, let us denote with $\varepsilon_{i}$ the observation of the phenomenon we intend to share (e.g. temperature) and with $x_{i}$ the random variable associated to the phenomenon we want to infer, (e.g. humidity).

If we associate each IoT device of our subnet with a random variable $X_{i}$, which represents the local information (such as temperature and humidity), the joint probability can be written as:

$$
P_{X}(x)=\prod_{i} \psi_{i}\left(x_{i}\right) \quad \prod_{i, j \in E} \psi_{i j}\left(x_{i}, x_{j}\right)
$$

where $\psi_{i}\left(x_{i}\right)$ is the evidence function, $E$ is the set of edges encoding the statistical dependencies between two nodes $i$ and $j$, and $\psi_{i j}()$ represents the potential function. Note that the graphical model parameters (i.e. $\psi_{i}$ and $\psi_{i j}$ ) can be estimated from the observed data by using a learning algorithm like in [8] and [7]. Figure 2 illustrates an example of the MRF model. The filled-in circles represent the observation nodes (i.e., $N_{\varepsilon_{i}}$ ) and the empty circles represent the hidden nodes $\left(\right.$ i.e., $\left.x_{i}\right)$. The potential functions are associated with the links between

\footnotetext{
${ }^{1}$ Clique is defined as a fully connected subset of nodes in the graph.
}

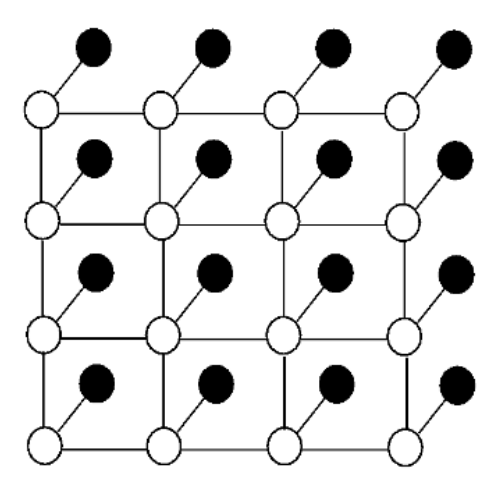

Figure 2: An example of MRF model.

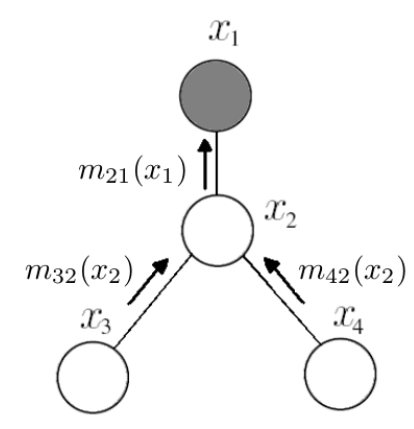

Figure 3: Message passing process in BP algorithm.

$x_{i}$ whilst the evidence functions are associated with the links between $N_{\varepsilon_{i}}$ and $x_{i}$.

We recall that $p\left(x_{i}\right)$ represents the marginal distribution of $i$-th node, and the BP allows the computation of $p\left(x_{i}\right)$ at each node $i$ by means of a message passing algorithm. The message from node $i$ to $j$ related to the local information $x_{i}$ is defined as:

$m_{j i}\left(x_{i}\right) \propto \int \psi_{j i}\left(x_{j}, x_{i}\right) \psi_{j}\left(x_{j}\right) \prod_{u \in \Gamma(j), u \neq i} m_{u j}\left(x_{j}\right) d x_{j}$,

where $\Gamma(j)$ denotes the neighbors of node $j$ and the incoming messages from previous iteration are represented by $m_{u j}$.

Eq. (4) will be performed between all nodes in the model until the convergence will be reached. Thus, the prediction i.e., the belief at each $i$-th node, is computed through all the incoming messages from the neighboring nodes and the local belief, i.e.:

$$
\hat{x_{i}}=\operatorname{belief}\left(x_{i}\right)=k \psi_{i}\left(x_{i}\right) \prod_{u \in \Gamma(i)} m_{u i}\left(x_{i}\right)
$$

where $k$ is a normalization constant.

It is worth to mentioning that the BP is able to compute the exact marginalization in the case of treestructured graphical models. Figure 3 illustrates the message passing process in BP. 


\section{EVALUATION \& DISCUSSION OF THE RESULTS}

\section{A. Experimental Setup}

In this section we provide the experimental results of our approach. Real data collected from 54 sensors deployed in the Intel Berkeley Research lab have been used. As mentioned before, these sensors collect temperature, humidity, light and voltage readings, as well as the network connectivity information which makes possible to reconstruct the network topology. Each data collection has been performed every 30 seconds. The original data consists of 38 days of readings. However, we will focus only on the first three hours of readings in this work. Figure 4 illustrates the scatterplot matrix of our data. In a nutshell, it shows the relationship between data during the first three hours of readings of the 54 sensors. We can notice that there is a good correlation between temperature and humidity data. Hence, we can easily infer the humidity data from temperature data and vice versa. In this paper, we decided to infer humidity from temperature. The temperature is in degrees Celsius, whilst the humidity is a value ranging from $0-100 \%$.

We assess our approach w.r.t. ( $i$ ) the number of transmitted data, (ii) the average value of the estimation error, (iii) the average value of the distortion level, and (iv) the energy consumption.

The number of transmitted data represents the total number of data transmission performed by all the sensors during the first three hours. The inference error is an important metric for any inference procedure. The goal is to have an errorless inference approach, i.e. an approach that is able to estimate the true value of data during all the inference procedures. However, this is almost never the case but we want that this error is as low as possible. In addition to the inference error, computing the distortion level is also important. This allows us to determine the difference between the real and the estimated value. The distortion level can be expressed using the Mean Squared Error (MSE) metric, which is defined as:

$$
M S E=\frac{1}{n} \sum_{i=1}^{n}\left(\hat{x}_{i}-x_{i}\right)^{2},
$$

where $\hat{x}_{i}$ and $x_{i}$ are respectively the predicted and true value during the $n$-th reading.

In our energy consumption evaluations, we assume that the energy cost for sending each temperature and humidity value is $14 \mathrm{~mW}$. This energy cost has been obtained on the Mica2Dot Berkeley mote as reported by [2]. Furthermore, all of our assessments are based on three different scenarios. In scenario $s 1$, the gateway sends to the cloud all the temperature and humidity data it receives. This means that the cloud does not perform any inference. In the second scenario $s 2$, the gateway sends only the temperature data to the cloud, and the cloud in turn infers the corresponding humidity data by using the BP algorithm. Finally, in the scenario $s 3$, we consider that the gateways are "smart" devices, meaning that before sending their data to the cloud, they first compute the probability $p(e \mid T, h)$ of making an inference error $e$ on the cloud given the temperature $T$, and the humidity $h$. If there is a strong chance that the error magnitude exceeds a predefined threshold, the gateway send both humidity and temperature data to the cloud, else the gateway send only the temperature data, and the

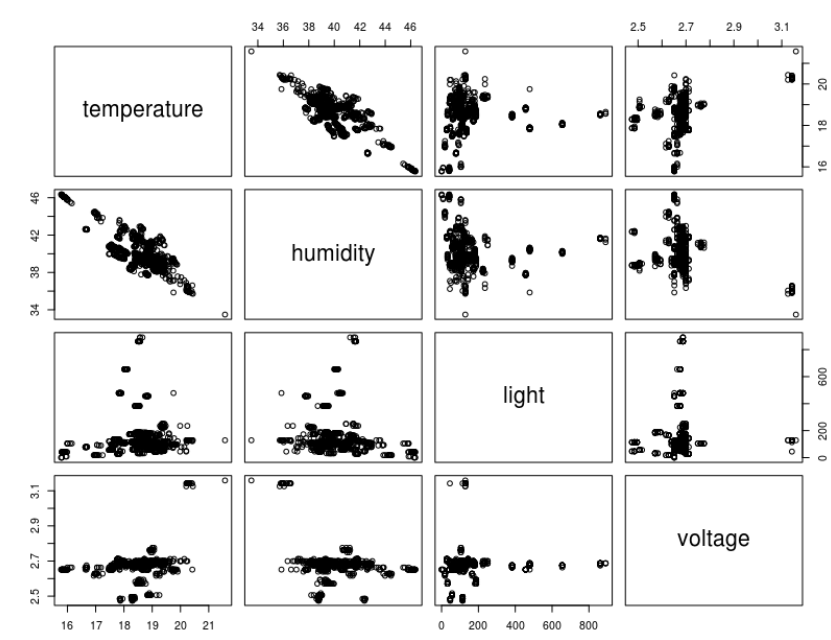

Figure 4: Correlation between data during the first three hours of readings.

\begin{tabular}{c|c|c|c|c}
\hline \hline Scenario & \#Transmitted data & $E C(\mathrm{~W})$ & $M S E$ & $E R$ \\
\hline \hline s1 & 20346 & 284.844 & - & - \\
\hline s2 & 10173 & 142.422 & 0.04 & 0.26 \\
\hline s3 & 12496 & 174.944 & 0.02 & 0.037 \\
\hline \hline
\end{tabular}

Table I: Results obtained during the first three hours of readings.

humidity value will be inferred in the cloud using the $\mathrm{BP}$ algorithm. The computation of $p(e \mid T, h)$ is done by means of the BP algorithm also. It should be noted that this computation requires the knowledge of the a priori probability of inference error, i.e., $p(e)$.

\section{B. Obtained results}

As mentioned before, we evaluate the performance of our approach in terms of number of transmitted data, average value of the estimation error (i.e., $E R$ ), average value of the distortion level (i.e., $M S E$ ), and energy consumption (i.e., $E C$ ). Our approach was implemented in $\mathrm{C}++$, and the assessments were performed with respect to the ground truth.

Table I illustrates the obtained results during the first three hours of readings. We can notice that our Bayesian inference approach drastically reduces the number of transmitted data and the energy consumption, while maintaining an acceptable level of prediction accuracy and information quality.

We can notice also that we decrease considerably the estimation error by using the scenario s3. Indeed, the gateways are smarter in this case. By computing the a posteriori probability of inference error, they will be able to estimate the right moment and the data type to send in the cloud. However, this increases the number of transmitted data (and hence the energy consumption), as compared to scenario s2. This is due to the fact that in s2 gateways send only the temperature data without worrying of the risk of inference error in the cloud.

Figure 5 shows the variation of the estimation error during the first three hours of reading. This corresponds 
to a MSE of 0.04 for scenario s2, and 0.02 for s3.

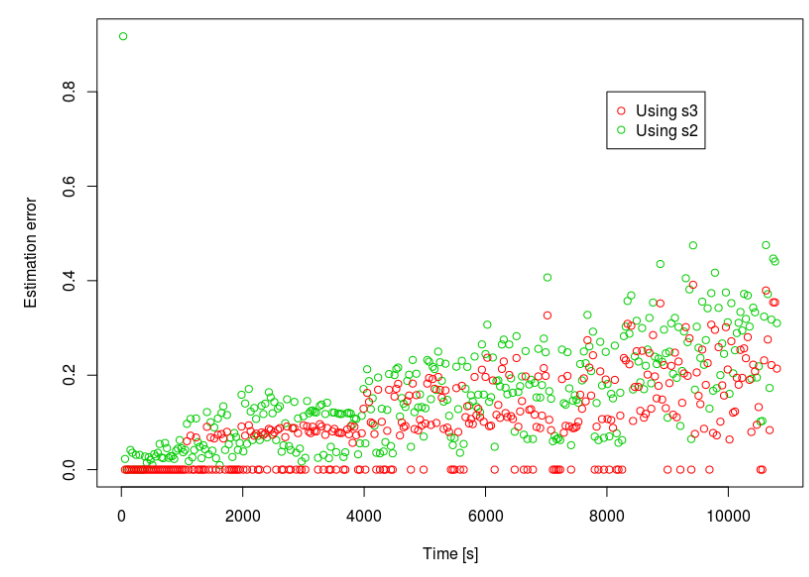

Figure 5: Variation of the estimation error.

\section{CONCLUSIONS}

In this paper, we have presented a inference-based approach that allows avoiding transmitting useless data in an heterogeneous IoT network. The strong correlation between data was taken into account for this study. Through extensive simulations and by using the real data collected from 54 sensors deployed in the Intel Berkeley Research lab, we have showed that our Bayesian inference approach reduces considerably the number of transmitted data and the energy consumption, while keeping an acceptable level of estimation error and information quality. We have also shown that the use of smart gateway decreases significantly the inference error. Note that even though we used a data source from WSN to validate our approach, it can be easily applied to different types of data sources provided by IoT devices. Future works will explore the possibility of doing the error prediction directly on the sensor nodes.

\section{ACKNOWLEDGMENT}

This work was partially supported by a grant from CPER Nord-Pas-de-Calais/FEDER Campus Intelligence Ambiante.

\section{REFERENCES}

[1] Giuseppe Anastasi, Marco Conti, Mario Di Francesco, and Andrea Passarella. Energy conservation in wireless sensor networks: A survey. Ad hoc networks, 7(3):537-568, 2009.

[2] Giuseppe Anastasi, Alessio Falchi, Andrea Passarella, Marco Conti, and Enrico Gregori. Performance measurements of motes sensor networks. In Proceedings of the 7th ACM international symposium on Modeling, analysis and simulation of wireless and mobile systems, pages 174-181. ACM, 2004.

[3] Mirza Mansoor Baig and Hamid Gholamhosseini. Smart health monitoring systems: an overview of design and modeling. Journal of medical systems, 37(2):1-14, 2013.
[4] Farshid Hassani Bijarbooneh, Wei Du, Edith C-H Ngai, Xiaoming $\mathrm{Fu}$, and Jiangchuan Liu. Cloud-assisted data fusion and sensor selection for internet of things. IEEE Internet of Things Journal, 3.

[5] Christopher M Bishop. Pattern recognition and machine learning. Machine Learning, 128, 2006.

[6] Council. Internet of things council. http://www. theinternetofthings.eu. Accessed November 18, 2015.

[7] Arthur P Dempster, Nan M Laird, and Donald B Rubin. Maximum likelihood from incomplete data via the em algorithm. Journal of the royal statistical society. Series B (methodological), pages 1-38, 1977.

[8] Zoubin Ghahramani. Graphical models: parameter learning. Handbook of brain theory and neural networks, 2:486-490, 2002.

[9] Caner Komurlu and Mustafa Bilgic. Active inference and dynamic gaussian bayesian networks for battery optimization in wireless sensor networks. In Proceedings of AAAI workshop on artificial intelligence for smart grids and smart buildings, 2016.

[10] Bin Liu, Zhenfeng Xu, Junjie Chen, and Geng Yang. Toward reliable data analysis for internet of things by bayesian dynamic modeling and computation. In Signal and Information Processing (ChinaSIP), 2015 IEEE China Summit and International Conference on, pages 1027-1031. IEEE, 2015.

[11] W. Hong S. Madden M. Paskin P. Bodik, C. Guestrin and R. Thibaux. Intel lab data. http://www.select.cs.cmu.edu/data/ labapp3/index.html. Accessed July 20, 2016.

[12] Riccardo Petrolo, Valeria Loscri, and Nathalie Mitton. Towards a smart city based on cloud of thoings. In Proceedings of the 2014 ACM international workshop on Wireless and mobile technologies for smart cities, WiMobCity, pages 61-66. ACM, 2014.

[13] Ou Qinghai, Zhen Yan, Li Xianghen, Zhang Yiying, and Zeng Lingkang. Application of internet of things in smart grid power transmission. In INFOCOM 2003. Twenty-Second Annual Joint Conference of the IEEE Computer and Communications. IEEE Societies, volume 2, pages 1293-1303. IEEE, 2003.

[14] Cristanel Razafimandimby, Valeria Loscri, and Anna Maria Vegni. A neural network and iot based scheme for performance assessment in internet of robotic things. In 2016 IEEE First International Conference on Internet-of-Things Design and Implementation (IoTDI), pages 241-246. IEEE, 2016.

[15] Surender Kumar Soni, Narottam Chand, and Dhirendra Pratap Singh. Reducing the data transmission in wsns using time series prediction model. In Signal Processing, Computing and Control (ISPCC), 2012 IEEE International Conference on, pages 1-5. IEEE, 2012.

[16] Liansheng Tan and Mou Wu. Data reduction in wireless sensor networks: A hierarchical lms prediction approach. IEEE Sensors Journal, 16(6):1708-1715, 2016.

[17] Anna Maria Vegni, Valeria Loscr, Alessandro Neri, and Marco Leo. A bayesian packet sharing approach for noisy iot scenarios. pages 305-308, 2016.

[18] Chaohui Wang, Nikos Komodakis, and Nikos Paragios. Markov random field modeling, inference \& learning in computer vision \& image understanding: A survey. Computer Vision and Image Understanding, 117(11):1610-1627, 2013.

[19] Mou Wu, Liansheng Tan, and Naixue Xiong. Data prediction, compression, and recovery in clustered wireless sensor networks for environmental monitoring applications. Information Sciences, 329:800-818, 2016.

[20] Jonathan S Yedidia, William T Freeman, and Yair Weiss. Understanding belief propagation and its generalizations. Exploring artificial intelligence in the new millennium, 8:236-239, 2003.

[21] Wei Zhao and Yao Liang. A systematic probabilistic approach to energy-efficient and robust data collections in wireless sensor networks. International Journal of Sensor Networks, 7(3):162$175,2010$. 